\title{
MAGNET FRINGE FIELDS, NONLINEAR EFFECTS, AND COMPENSATION IN LARGE ACCEPTANCE RINGS
}

\author{
K. Makino*, Department of Physics, University of Illinois at Urbana-Champaign, \\ Urbana, IL 61801, USA \\ B. Erdélyi ${ }^{\dagger}$, M. Berz ${ }^{\ddagger}$, Department of Physics and Astronomy and NSCL, \\ Michigan State University, East Lansing, MI 48824, USA
}

\begin{abstract}
The importance of nonlinear effects has been well known in the field of modern high resolution spectrography and in other areas requiring the precise manipulation of large phase space volumes. Simulations for such devices require treating the field as precisely as possible; furthermore the required large aperture of the appearing magnets usually makes the consideration of fringe fields crucial.

In conventional proton beam rings, many of the nonlinear effects can often be safely neglected because of the relatively small emittances. This is not the case anymore in large acceptance rings such as those to be used for the various muon accelerator scenarios, where the difficulty and expense of cooling requires the ability to manipulate a beam of unusually large emittance.

We will discuss various nonlinear effects including those due to fringe fields and the so-called kinematic correction for proposed muon storage rings, and present novel methods of symplectic tracking and other nonlinear analysis tools as well as schemes to compensate the nonlinear effects.
\end{abstract}

\section{INTRODUCTION}

Conventionally, various nonlinear effects are often neglected in simulations of large storage rings. In most cases, it is a safe assumption for small emittance beams, and this has considerably simplified the simulation of storage ring dynamics. As it will be shown in this paper, fringe field effects affect the linear and nonlinear motion of beams. If the magnet aperture is large, the sharp cut-off approximation is unrealistic and furthermore practically the fringe fields tend to be more complicated. As the consequence, even the kick approximation is often not sufficient, and complicated higher order effects are incurred. Since the nonlinear effects are larger for beams with larger emittance, those effects are especially crucial for devices with large acceptance.

Not only large acceptance spectrographs, but also some storage ring designs encounter such a situation. One case is

\footnotetext{
*makino@uiuc.edu, the work was supported by the Illinois Consortium for Accelerator Research under contract FY-2001-IBHE-HECA and the National Science Foundation.

† erdelyib@msu.edu

$\ddagger$ berz@msu.edu, the work was supported by the US Department of Energy under contract DE-FG02-95ER40931, an Alfred P. Sloan Fellowship and the National Science Foundation.
}

the need of strong focusing of beams near a collider interaction region to achieve high luminosity. The large space for detectors around the interaction point forces to have a longer drift in the optics, resulting in large aperture magnets for the final strong focusing. We performed a study for the LHC, and found noticeable fringe field effects $[1,2]$. Another case is muon beam storage rings, where the difficulty and expense of beam cooling leave the emittance large. Studies of some proposed muon storage rings indicate in fact that nonlinear effects are not negligible any more and great care has to be taken. In this paper, we will use a lattice of a proposed neutrino factory, a $30 \mathrm{GeV}$ neutrino factory ring lattice kindly supplied by C. Johnstone [3], as an example to review the various nonlinear effects. For more systematic and detailed studies and discussions, refer to [4].

In the next section, we will start the discussion on the so-called kinematic correction. Despite of the simplicity of the concept and procedure as will be discussed, the kinematic correction is not so widely employed. The effect is observed from the second order [5], and as will be shown, an idea to bring the next lowest order in consideration is not good enough and would even lead to a faulty conclusion. The discussion of the fringe field effects follows in the subsequent section. We will demonstrate the magnet aperture dependence of the linear tunes. By identifying the most influential magnets, the suppression of the effects can be manipulated. At a more advanced study level, details of the magnet fringe fields are an issue to be addressed. Using various shapes of the fall-off of the magnet fringe fields, we will also demonstrate how the beam dynamics depends on the details of the magnet fringe fields.

\section{THE KINEMATIC CORRECTION}

The most general form of the Hamiltonian of a charged particle in electromagnetic fields in curvilinear coordinates $\{s, x, y\}$ can be written in terms of the generalized momentum $\vec{P}^{G}=\left(P_{s}^{G}, P_{x}^{G}, P_{y}^{G}\right)$ as [6, 7]

$$
\begin{aligned}
H= & q \Phi+c\left[\frac{\left(P_{s}^{G}+P_{x}^{G} \tau_{1} y-P_{y}^{G} \tau_{1} x-\alpha q A_{s}\right)^{2}}{\alpha^{2}}\right. \\
& \left.+\left(P_{x}^{G}-q A_{x}\right)^{2}+\left(P_{y}^{G}-q A_{y}\right)^{2}+m^{2} c^{2}\right]^{1 / 2}
\end{aligned}
$$

Here, $\tau_{1}$ is the rate of rotation around the beam axis, $\tau_{2}$ and $\tau_{3}$ are curvatures in $y$-s and $x$-s planes, and $\alpha$ is de- 
fined as $\alpha=1-\tau_{3} x+\tau_{2} y$. The generalized momentum is expressed in terms of the kinematic momentum $\vec{p}=\left(p_{s}, p_{x}, p_{y}\right)$, where we usually expect $p_{s}^{2} \gg p_{x}^{2}, p_{y}^{2}$, as

$$
\begin{gathered}
P_{s}^{G}=\left(p_{s}+q A_{s}\right) \alpha-\left(p_{x}+q A_{x}\right) \tau_{1} y+\left(p_{y}+q A_{y}\right) \tau_{1} x \\
P_{x}^{G}=p_{x}+q A_{x}, \quad P_{y}^{G}=p_{y}+q A_{y} .
\end{gathered}
$$

Apparently the first term in the square root in the Hamiltonian (1) is the leading term, and the approximation to omit the second and the third terms, which entail nonlinear effects purely due to dynamics and independent of the fields, is widely used. In case of large transversal emittances, $p_{x}$ and $p_{y}$ are not small compared to $p_{s}$ anymore. The restoration of those omitted terms is often referred to as kinematic correction. Effects on the dynamics by the kinematic correction appear typically from second order effects for the horizontal motion, and third order effects for the vertical motion [5].

The code COSY INFINITY is a transfer map based beam physics code working to arbitrary high order $[8,9$, 10]. All nonlinear terms are included in the necessary equations [8] from the outset, and the nonlinear effects can be taken care of up to any desired order. Thus, the code includes kinematic correction without any approximation by using the exact expression for the dynamics [5]. In order to study the effects of kinematic correction, which is frequently omitted in other codes, options were created that selectively ignore $p_{x}^{2}$ and $p_{y}^{2}$ compared to $p_{s}^{2}$ (the zero-th order approximation), or take the next lowest consideration of $p_{x}^{2}$ and $p_{y}^{2}$ (the second order approximation).

As an example, we review the effects on a neutrino factory ring $[5,3]$. We studied the effect on the size of dynamic aperture by tracking particles via 7 th order transfer maps. In case of no kinematic correction (the zeroth order approximation), particles with $x$ and $a=p_{x} / p_{0}$ up to $1000 \mathrm{~mm} \times 150 \mathrm{mrad}$ are stable in the ring. However when the full kinematic correction is included, the particles with $x \times a$ up to $100 \mathrm{~mm} \times 15 \mathrm{mrad}$ are stable, decreasing the dynamic aperture by a factor of 100. Fig. 1 illustrates how the particles are preserved under the influence of the kinematic correction by tracking pictures in a scale of $500 \mathrm{~mm} \times 60 \mathrm{mrad}$. The first picture (A) shows the motion without kinematic correction (the zero-th order approximation), where the motion seems very stable. The second picture (B) shows with full kinematic correction, which is the default way in COSY INFINITY, showing the significant decrease in the dynamic aperture, and it would present a difficulty if the acceptance is larger than $10 \mathrm{~cm}$. We also studied the effect of the next lowest order kinematic correction (the second order approximation) because it is sometimes believed that the next lowest order correction is enough, and the result is shown in the picture (C). To confirm the result, we applied the optimal generating function symplectification, EXPO [11], as shown in the last picture (D). The further loss of the dynamic aperture by the second order approximation tells us that one should

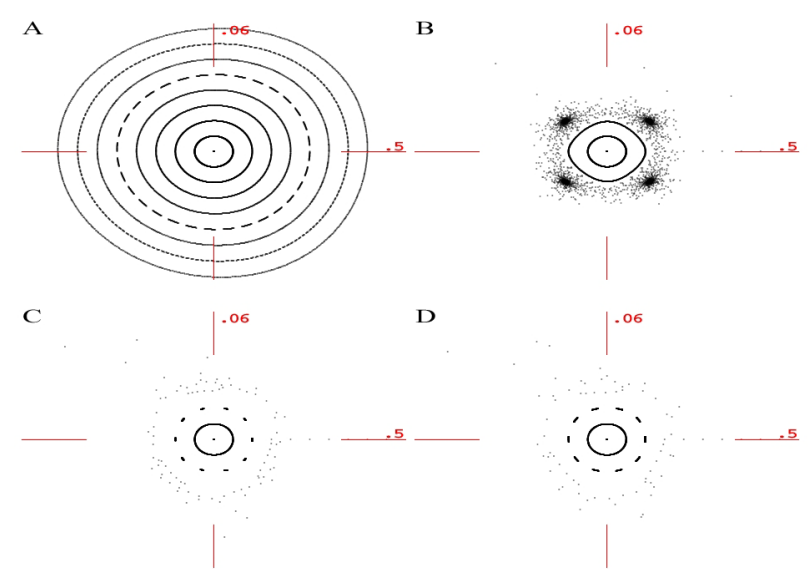

Figure 1: Tracking pictures in the horizontal phase space in a $30 \mathrm{GeV}$ neutrino factory ring in a scale of $500 \mathrm{~mm} \times 60 \mathrm{mrad}$. A) Without kinematic correction. B) With full kinematic correction. C), D) With the next lowest order kinematic correction (the second order approximation) without (C) and with (D) the EXPO symplectification [11].

use the full correction that requires the treatment of square roots, instead of the approximation that consists of additions of squares. The amount of the effects is compared to the fringe field effects in Fig. 2 in the next section, and the effects by the kinematic correction are not so dramatic under the influence of the fringe field effects.

\section{FRINGE FIELD EFFECTS}

For simulations of large storage rings, fringe field effects are often neglected. Even though sometimes this is a quite good approximation, strictly speaking, it is an unphysical model, as the electromagnetic fields of the model do not satisfy Maxwell's equations. The simplest method to take fringe fields into account is to take their effect with a kick characterized by the integrated field value [12], but more sophisticated models are needed for accurate simulations. Refer to [4] for the detailed discussion on the sharp cut-off approximation, and various studies on fringe field treatments. In the map picture using Differential Algebraic methods $[13,14,15]$, the fringe field effects can be particularly easily studied. The fringe field effects influence all orders of the motion, beginning with the linear behavior, and the complete treatment to any order is possible in the code COSY INFINITY $[8,9,10]$.

The general solution of the Laplace equation in cylindrical coordinates is

$$
V=\sum_{k, l=0}^{\infty}\left(b_{k, l}(s) \sin l \phi+a_{k, l}(s) \cos l \phi\right) r^{k} .
$$

The functions $b_{l, l}(s)$ are the normal and $a_{l, l}(s)$ the skew multipoles, and $l=0,1,2,3, \ldots$ correspond to the solenoid, dipole, quadrupole, sextupole,... components. They induce 


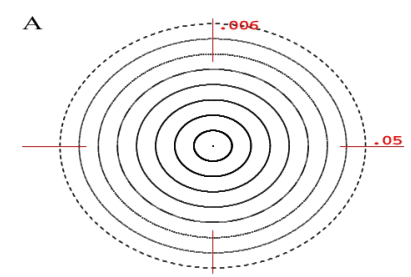

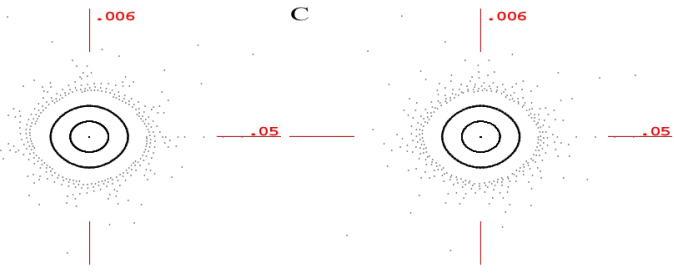

Figure 2: Tracking pictures in a scale of $50 \mathrm{~mm} \times 6 \mathrm{mrad}$. A) Without fringe field effects. B), C) With fringe field effects, and further without (B) and with (C) kinematic correction. The aperture of all the magnets is $50 \mathrm{~mm}$, and COSY's default Enge fall-off shape is used for the fringe fields.

the following recursion relations [15].

$$
\begin{aligned}
& b_{l+2 n, l}(s)=\frac{b_{l, l}^{(2 n)}(s)}{\Pi_{\nu=1}^{n}\left(l^{2}-(l+2 \nu)^{2}\right)} \\
& a_{l+2 n, l}(s)=\frac{a_{l, l}^{(2 n)}(s)}{\Pi_{\nu=1}^{n}\left(l^{2}-(l+2 \nu)^{2}\right)} .
\end{aligned}
$$

Sometimes they are called pseudo-multipoles, and the only free parameters in the general form of the potential are the $s$-dependent multipoles $a_{l, l}(s)$ and $b_{l, l}(s)$. For practical calculations, these are either fitted to represent measured data, or obtained by multipole decomposition of detailed field computations [11]. The additional pseudo-multipole nonlinearities of the fringe fields couple to higher derivatives of the multipole strengths. In practice this entails that fringe field effects become more and more relevant the more the particles are away from the axis of the elements, which is directly connected to the emittance of the beam. The fall-off shapes, and implicitly the apertures, have an influence on the induced nonlinearities, too.

We use the neutrino factory ring to illustrate general trends in large acceptance rings. The ring is at a preliminary design stage so no actual magnet configuration is available yet. We take the fall-off shape of the fringe fields modeled by a six parameter Enge function, which depends on the size of the magnet aperture. To get a rough idea comparable to the kinematic correction study in the previous section, particles are tracked using COSY INFINITY assuming COSY's default fringe field shape [16] and the (half) aperture of $50 \mathrm{~mm}$. The first picture (A) in Fig. 2 is just the same with the picture (B) in Fig. 1, with full kinematic correction, but in a magnified scale. There are no fringe field effects included. The other pictures in Fig. 2 are under the influence of the fringe field effects, and further without (B) and with (C) kinematic correction. With fringe field effects, a further decrease in the dynamic aper-

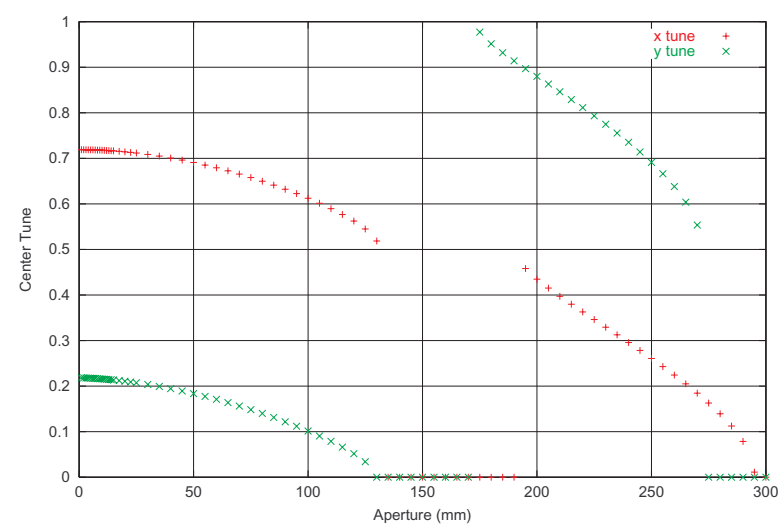

Figure 3: Center tunes as a function of aperture. COSY's default Enge fall-off shape is used for the fringe fields.

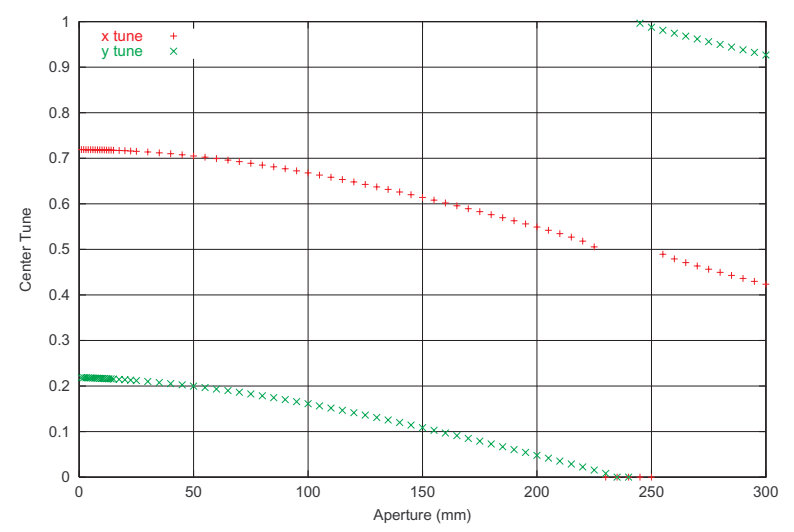

Figure 4: Center tunes without the matching section, where most of the fringe field effects are concentrated.

ture by a factor of 100 is observed, but there is no significant difference by the kinematic correction at this level.

\subsection{Aperture Dependent Effects on Linear Tunes}

We look at fringe field effects as a function of magnet aperture and fall-off shape, which is achieved by varying the size of the magnet apertures and the six Enge coefficients. We compute the linear tunes as a function of the magnet aperture. We assume that all the magnets have the same aperture, and the fall-off is given by COSY's default Enge coefficients. Roughly speaking, magnets with larger aperture extend the fringe field region, thus larger fringe field effects are expected. Frequently the Enge function model can be used for a global fit of the magnetic fields, including the out of axis expansion. This has been demonstrated in several real situations, as for example NSCL's S800 spectrograph [17], the GSI QD kaon spectrometer [18], and even the rather peculiar Large Hadron Collider's High Gradient Quadrupole (LHC HGQ) lead end $[19,1]$.

The half aperture is varied between $1 \mathrm{~mm}$ and $300 \mathrm{~mm}$. Fig. 3 shows the results for the $x$ and $y$ center tunes. In the 

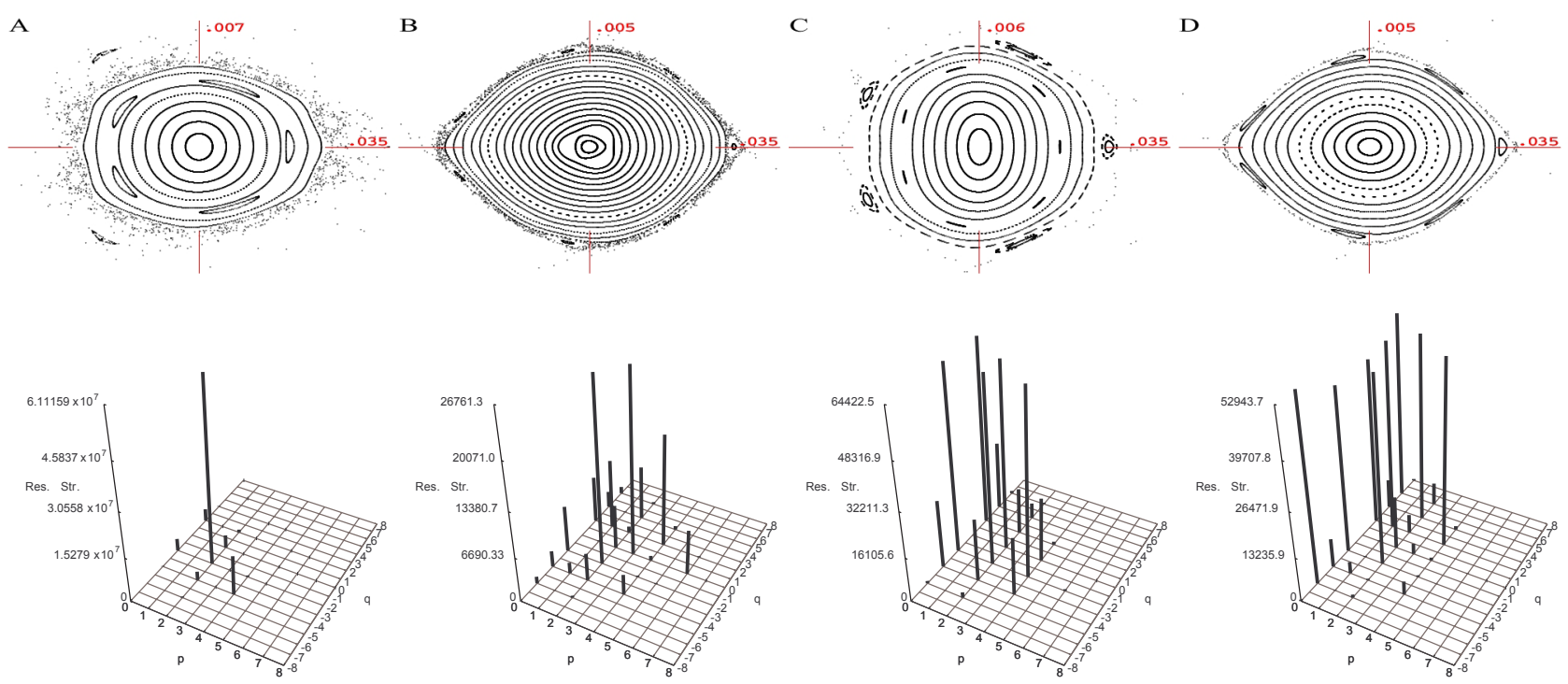

Figure 5: Tracking pictures in scales of $35 \mathrm{~mm} \times 5-7 \mathrm{mrad}$, and resonance strengths along the diagonal in action space. The magnet half aperture is $75 \mathrm{~mm}$ for all four types of fringe field fall-off; A) COSY's default dipole, B) COSY's default quadrupole, C) LHC HGQ lead end, and D) GSI QD spectrograph.

stable regions the tunes change continuously and monotonically with the aperture.

It has been noticed that the main impact of the fringe field effects comes only from a few matching quadrupoles in the arcs [20]. We performed the computations of the ring, with the respective matching quadrupole fringe fields turned off, and the results are shown in Fig. 4. As expected, the aperture dependence of the center tunes is weaker. In such a way, we can concentrate the study on those influential magnets to manipulate the amount of the effects.

\subsection{Shape Dependent Effects}

Here we study the influence of the fall-off shape. We prepare several different sets of Enge coefficients only for the quadrupoles in the same lattice. We review four cases in this paper; (A) COSY's default dipole magnet fall-off; (B) COSY's default quadrupole magnet fall-off [16]; (C) a lead end of the LHC HGQs in the interaction regions $[19,1]$; and (D) a warm, large (half) aperture $(\sim 15 \mathrm{~cm})$ quadrupole in a QD kaon spectrometer at GSI [18]. Those sets were taken from fitting measured or simulated magnetic field data, and the fits represent the fields globally as well as along the optical axis.

The tune shifts and resonance strengths via normal form methods [15], and the dynamic apertures by tracking were studied for several aperture size cases [4]. We used 8th order transfer maps for the tracking, employing the optimal generating function symplectification, EXPO [11]. We show the dynamic aperture and the resonance strengths pictures in Fig. 5 for the $75 \mathrm{~mm}$ (half) aperture case. The resonance strengths have been calculated along the diagonal in action space, at a value that approximately corresponds to the dynamic aperture. In the results, there is no really good correlation between the three different quantities computed [4]. For example, the lattice with dipole type fringe field has larger than average amplitude dependent tune shifts and resonance strengths, which results in a smaller dynamic aperture. On the other hand, the LHC HGQ type fringe fields result in even larger tune shifts, but the tracking shows a relatively clean looking phase space with an average dynamic aperture. This indicates that it is wise to study fringe field effects on a case-by-case basis.

\section{CONCLUSIONS}

We reviewed kinematic correction and fringe field effects as major nonlinear effects from the theoretical point of view and in simulations. Those effects are more crucial in large acceptance rings, and we reviewed the fringe field effects depending on various factors, namely the size of magnet aperture and the fringe field fall-off shape. The more detailed study on them is discussed in [4].

The results of the study on the kinematic correction suggest to use the full kinematic correction for large acceptance rings. The results on the fringe field effects indicate that one should study the effects even in the beginning design stage for large acceptance rings. Some influential magnets could be identified to manipulate the amount of the effects at an earlier design stage. When the design progresses, one should study detailed effects based on the actual magnet design and, if available, the measurement.

Both the kinematic correction and the fringe field effects can be taken into account straightforwardly in the code COSY INFINITY, which is based on the transfer map approach utilizing the Differential Algebraic method. When studying the dynamic aperture by tracking via transfer maps, it is important to be able to have an appropriate 
symplectification. A new approach, EXPO, employs the optimal generating function for symplectification [11].

\section{REFERENCES}

[1] W. Wan, C. Johnstone, J. Holt, M. Berz, K. Makino, and M. Lindemann, "The Influence of Fringe Fields on Particle Dynamics in the Large Hadron Collider", Nuclear Instruments and Methods, A427:74-78, 1999.

[2] B. Erdélyi, M. Berz and K. Makino, "Detailed Analysis of Fringe Field Effects in the Large Hadron Collider", Technical Report MSUCL-1129, National Superconducting Cyclotron Laboratory, Michigan State University, East Lansing, MI 48824, USA, 1999.

[3] C. Johnstone, Private communication.

[4] M. Berz, B. Erdélyi, and K. Makino, "Fringe Field Effects in Small Rings of Large Acceptance", Physical Review STAB, 3:124001, 2000.

[5] K. Makino and M. Berz, "Effects of Kinematic Correction on the Dynamics in Muon Rings", AIP CP, 530:217-227, 2000 .

[6] K. Makino and M. Berz, "Pertubative Equations of Motion and Differential Operators in Nonplanar Curvilinear Coordinates", International Journal of Applied Mathematics, 3,4:421-440, 2000.

[7] M. Berz and K. Makino, "Preservation of Canonical Structure in Nonplanar Curvilinear Coordinates", International Journal of Applied Mathematics, 3,4:401-419, 2000.

[8] M. Berz, "Computational Aspects of Design and Simulation: COSY INFINITY”, Nuclear Instruments and Methods, A298:473, 1990.

[9] K. Makino and M. Berz, "COSY INFINITY Version 8”, Nuclear Instruments and Methods, A427:338-343, 1999.
[10] M. Berz, "COSY INFINITY Version 8 Reference Manual", Technical Report MSUCL-1088, National Superconducting Cyclotron Laboratory, Michigan State University, East Lansing, MI 48824, USA, 1997. See also http://cosy.nscl.msu.edu.

[11] B. Erdélyi, "Symplectic Approximation of Hamiltonian Flows and Accurate Simulation of Fringe Field Effects", $\mathrm{PhD}$ thesis, Michigan State University, East Lansing, Michigan 48824, USA, 2001.

[12] G. Lee-Whiting, Nuclear Instruments and Methods, 83:232, 1970.

[13] M. Berz, "Arbitrary Order Description of Arbitrary Particle Optical Systems", Nuclear Instruments and Methods, A298:426, 1990.

[14] M. Berz, "Differential Algebraic Description of Beam Dynamics to Very High Orders", Particle Accelerators, 24:109, 1989.

[15] M. Berz, "Modern Map Methods in Particle Beam Physics", Academic Press, San Diego, 1999.

[16] K. L. Brown and J. E. Spencer, "Non-linear Optics for the Final Focus of the Single-pass-collider", IEEE Transactions on Nuclear Science, NS-28,3:2568, 1981.

[17] D. Bazin, Private communication, 2000.

[18] F. Meot, Private communication, 2000.

[19] G. Sabbi, "Magnetic Field Analysis of HGQ Coil Ends", Technical Report TD-97-040, Fermilab, 1997.

[20] F. Zimmermann, C. Johnstone, M. Berz, B. Erdélyi, K. Makino, and W. Wan, "Fringe Fields and Dynamic Aperture in Muon Storage Rings", Technical Report 95, Muon Collider Collaboration, BNL, 2000. 